\title{
Deterministic spin models with a glassy phase transition
}

\author{
I.Borsari, M.Degli Esposti, S.Graffi and F.Unguendoli \\ Dipartimento di Matematica, Università di Bologna \\ 40127 Bologna, Italy
}

\begin{abstract}
We consider the infinite-range deterministic spin models with Hamiltonian $H=\sum_{i, j=1}^{N} J_{i, j} \sigma_{i} \sigma_{j}$, where $J$ is the quantization of a chaotic map of the torus. The mean field (TAP) equations are derived by summing the high temperature expansion. They predict a glassy phase transition at the critical temperature $T \sim 0.8$.
\end{abstract}

PACS N. 64.60 Cn, 64.60 Fr, 64.70 Pf

A class of infinite-range deterministic Ising spin models with glassy behaviour in numerical simulation has been recently identified [1], [2], [3]: unlike the random coupling case, however, the mean field equations [四] (hereafter the PP equations) are different from the standard TAP ones [5] and their linearization does not determine a critical temperature for the glassy transition.

The most interesting representatives of the class are the sine (equivalently, cosine) models, with Hamiltonian

$$
H=-1 / 2 \sum_{i, j=1}^{N} J_{i, j} \sigma_{i} \sigma_{j}
$$

where the coupling matrix $J$ is (twice the uppermost left block) of the discrete sine (cosine) Fourier transform

$$
J_{i, j}=\frac{2}{\sqrt{2 N+1}} \sin \left(\frac{2 \pi i j}{2 N+1}\right), \quad i, j=1, \ldots, N
$$

In fact, the ground state of $H$ can be explicitly computed [3] if $2 N+1$ is prime with $N$ odd: this leads to detect a first-order crystalline phase transition (at a higher critical temperature than the glassy one [2, 3]), whose relevance on the glassy behaviour of the system is discussed in [4], §3. In turn, the matrix $J$ coincides [6] with (the imaginary 
part of) the unitary propagator quantizing the discrete dynamics generated by the unit symplectic matrix $S=\left(\begin{array}{cc}0 & -1 \\ 1 & 0\end{array}\right)$ acting as a Hamiltonian map over the 2 -torus $\mathbb{T}^{2}$ (the operator quantizing a Hamiltonian map of the torus is a $N \times N$ unitary matrix [7,8], $N$ being the inverse of the Planck constant:" in this context the thermodynamic limit $N \rightarrow \infty$ is formally equivalent to the classical limit). This algebraic identity suggests as more natural candidates for detecting deterministic glassy behaviour the coupling matrices defined by the quantization of hyperbolic maps over $\mathbb{T}^{2}$. The corresponding discrete dynamical systems are indeed chaotic, while $S$ generates a period-4 one. Here we consider the matrices $A=\left(\begin{array}{ll}a & b \\ c & d\end{array}\right)$ with $a=d=2 g, b=1, c=4 g^{2}-1, g \in \mathbb{N}$, which admit two positive eigenvalues $\lambda_{1}>1, \lambda_{2}<1$. The quantization of $A$ is [7] [9] the unitary $N \times N$ matrix

$$
V(A)_{j k}=C_{N} N^{-1 / 2} \exp \left[(2 \pi i / N)\left(g j^{2}-j k+g k^{2}\right)\right]
$$

with $\left|C_{N}\right|=1$. The present models are thus defined by the Hamiltonians $H_{A}(\sigma)=$ $\sum_{j, k} J(A)_{j k} \sigma_{j} \sigma_{k}$ with $J(A)=\operatorname{Re}[V(A)]$, i.e.:

$$
J(A)_{j k}=C_{N} N^{-1 / 2} \cos \left[(2 \pi i / N)\left(g j^{2}-j k+g k^{2}\right)\right]
$$

Now $\bar{V}(A)=\bar{V}^{T}(A)=V^{-1}(A)$ whence $20(\operatorname{Re}[V(A)])=\operatorname{Re}[\sigma(V(A))]$. Hence the equistribution [10] of the spectrum $\sigma(V(A))$ over the unit circle for $N \rightarrow \infty$ with an eigenvalue at 1 for all $N$ implies the same properties for $\sigma(J(A))$ in $[-1,1]$.

Our claim is that these deterministic models behave, at least in mean field approximation, more closely to the random ones, such as the SK one [11]. We will indeed

\footnotetext{
${ }^{1}$ The physical intuition is: the phase space has volume 1 , and can accommodate at most $N$ quantum states of volume $\hbar$, so that $N \hbar=1$.

${ }^{2}$ This holds only if $a, b, c, d$ are as above, and motivates our choice of hyperbolic matrices.
} 
compute in closed form (at the thermodynamic limit $N \rightarrow \infty$ ) the Gibbs (i.e., magnetization dependent) free energy $\beta \Phi$. The result is:

$$
\begin{gathered}
\beta \Phi=1 / 2 \sum_{i=1}^{N}\left\{\left(1+m_{i}\right) \ln \left(1+m_{i}\right) / 2+\left(1-m_{i}\right) \ln \left(1-m_{i}\right) / 2\right\}- \\
-\beta / 2 \sum_{i, j=1}^{N} J(A)_{i j} m_{i} m_{j}-N G(\beta(1-q)) ; G(\beta)=\beta^{2} /\left(8+4 \beta^{2}\right)
\end{gathered}
$$

where $m_{l}: l=1, \ldots, N$ is the magnetization at site $l$ and $q=1 / N \sum_{l=1}^{n} m_{l}^{2}$ the EdwardsAnderson order parameter (as in [4], $G(\beta)$ does not depend on the particular choice of $J(A))$. The stationarity condition of the Gibbs free energy [12 yields the mean field equations of the model (believed exact because of its infinite range)

$$
Q_{i} \equiv \tanh ^{-1} m_{i}+2 \beta G^{\prime}(\beta(1-q)) m_{i}-\beta \sum_{j} J(A)_{i j} m_{j}=0
$$

Unlike the PP ones (but like the TAP [5]) these equations can be solved by linearization near $q=0$ : any eigenvector for the eigenvalue 1 of $J(A)$ yields a solution if $2 \beta^{2} /\left(2+\beta^{2}\right)^{2}-\beta+1=0$. Its only positive zero $\beta \sim 1.25$ determines the critical temperature $T \sim 0.8$. The phase transition is glassy because, as we will see, $1 / N \sum_{l=1}^{N} m_{l} \rightarrow 0$ as $N \rightarrow \infty$. This fact and (5) implies that all such solutions have the same free energy. The thermodynamics is thus independent of their number, the multiplicity of the eigenvalue 1, which depends in a sensitive way on the integer $N$ [10].

To get the mean field equations (6) we resum the high temperature expansion by the same procedure of $[4$. The function $G(\beta)$ has a simpler form in this case because there is only one class of non vanishing diagrams at the thermodynamic limit.

Consider the Helmholtz free energy $F(\beta)$ : if $Z(\beta)=\sum_{\{\sigma\}} \exp \beta H_{A}(\sigma) / 2=\exp -\beta F(\beta)$ is the partition function (at site-dependent magnetic field $h_{i}=0$ ) we have [13

$$
\begin{gathered}
e^{-\beta F}=(2 / \pi)^{N / 2} /\left.\operatorname{det}^{\frac{1}{2}}(\beta J) \int_{\mathbb{R}^{N}} \exp \left\{\left\langle(2 \beta J)^{-1} \phi, \phi\right\rangle+\sum_{i} \log \cosh \left(\phi_{i}+h_{i}\right)\right\} d^{N} \phi\right|_{h_{i}=0}= \\
2^{N} \pi^{-N / 2} \int_{\mathbb{R}^{N}} \exp \left\{-<x, x>+\sum_{i=1}^{N} \log \cosh \left[\sqrt{2 \beta} \sum_{h}\left(\left(J^{1 / 2}\right)_{i h} x_{h}\right]\right\} d^{N} x\right.
\end{gathered}
$$


The high temperature expansion for $\beta F(\beta)$ is generated out of the integration of the expansion of $\exp \log \cosh x$. The well known diagrammatic representation of its $n$-th order term is obtained [13] by drawing all diagrams with $n$ links, $2 \leq j+1 \leq n+1$ vertices and no external legs, whose individual contribution is

1. For any link between two consecutive vertices $l \neq k$ a factor $\beta J_{l k}$;

2. For any vertex with $m$ links the cumulant $u_{m}$, i.e. the $m$-th coefficient of the Taylor expansion of $\log \cosh x($ [13], p.414);

3. Any diagram has to be divided by its order of symmetry.

The contribution of each individual diagram $D$ at order $\beta^{n}$ is indeed

$$
|D|=U(D) S(D)^{-1} \sum_{r_{1}, \ldots, r_{j+1}} J_{r_{1}, r_{2}}^{\alpha_{1}} \cdot J_{r_{2}, r_{3}}^{\alpha_{2}} \cdots J_{r_{j}, r_{j+1}}^{\alpha_{j}}, \quad j=1, \ldots, n
$$

Here $j+1$ is the number of vertices, $\alpha_{1}+\ldots+\alpha_{j}=n ; n-j+1=n-1, \ldots, 1$ is the number of loops, $\alpha_{i} \geq 1$ the number of links between consecutive vertices, $S(D)$ the symmetry factor, and $U(D)=u_{1}\left(\alpha_{1}\right) u_{2}\left(\alpha_{1}+\alpha_{2}\right) \cdots u_{j}\left(\alpha_{j-1}+\alpha_{j}\right) u_{j+1}\left(\alpha_{j}\right)$. Remark that $r_{n+1}=r_{1}$ for $j=n$. We now verify that, at the thermodynamic $\operatorname{limit} N \rightarrow \infty$ :

(a) All diagrams for $n=2 p+1$ vanish;

(b) For $n=2 p$ the only surviving diagram $D_{p}$ is the one with $p+1$ vertices, $p$ loops and two links between consecutive loops:

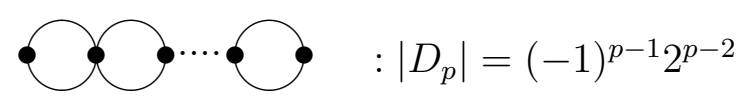

To prove (a) and (b), first recall the basic estimate fulfilled by the Gauss sums [14

$$
\left|\sum_{s_{1}, \ldots, s_{l}=1}^{N} \exp (2 \pi i / N) g\left(s_{1}, \ldots, s_{l}\right)\right| \leq C N^{l / 2}
$$

where $g$ is any quadratic form in the $l$ integers $s_{1}, \ldots, s_{l}$ with integer coefficients and $C$ a constant independent of $g$ and $N$. By (3) and (4), the sum in (8) amounts for any fixed $1 \leq j \leq n$ to $2^{n}$ Gaussian sums over $r_{1}, \ldots, r_{j}$ divided by $N^{n / 2}$. We now argue that all these Gaussian sums are, once divided by $N^{n / 2}$, of order 1 or less except one: that with $n=2 p, \alpha_{1}=\ldots=\alpha_{p}=2$, and summand independent of $r_{1}, \ldots, r_{p+1}$, 
generated by the constant term in the expansion of $\prod_{k} J_{r_{k}, r_{k+1}}^{2}$. This sum is clearly equal to $N^{p+1} N^{-p} 2^{-p}=N 2^{-p}$. Any other (divided) sum is down by at least $N^{-1}$ : each summation over $q$ indices is estimated by $N^{q / 2}$ if the summand is a Gaussian and by $N^{q}$ if the summand is constant, which case shows up whenever $\alpha_{i}$ is even for at least one $i$; on the other hand any power of $\alpha_{i}$ in excess of one increases by one the number of loops and thus reduces by one the number of vertices and hence of summation indices. Thus for $n=2 p+1$ the most divergent sums behave as $N^{p+1 / 2}$ : this happens either for $\alpha_{i}=1$ for all $i$ so that $j=2 p$, or for $\alpha_{1}=\ldots=\alpha_{p-1}=2, \alpha_{p}=3$ (and permutations thereof) so that $j=p+1$. The prefactor $N^{-(p+1 / 2)}$ yields the estimate $O(1)$. For $n=2 p$ the second most divergent sums have the same behaviour: again they take place for $\alpha_{i}=1$ for all $i$ and $j=2 p-1$ or for $\alpha_{1}=\ldots=\alpha_{p}=2$ with the summand depending but on two indices (up to permutations). Now the only surviving $D_{p}$ has two vertices with two links (the extrema) and $p-1$ vertices with four links (all the remaining ones). Since $u_{2}=1, u_{4}=-2, U\left(D_{p}\right)=(-2)^{p-1}$. Moreover the symmetry factor $S\left(D_{p}\right)$ is $2 \cdot 2^{p}$ (to account for the interchange of the external vertices and of any pair of links between the internal vertices). Hence $U\left(D_{p}\right) / S\left(D_{p}\right)=(-1)^{p-1} / 4$ and by (8) we have $\left|D_{p}\right|=(-1)^{p-1} \cdot 2^{-p-2}$. Summing up we get the Helmholtz free energy:

$$
-\beta F(\beta)-N \ln 2=N \sum_{p=1}^{\infty}(-1)^{p-1} \cdot 2^{-p-2} \beta^{2 p}=N \beta^{2} /\left(8+4 \beta^{2}\right)=N G(\beta)
$$

As in [⿴囗十), expanding $-\beta F(\beta)$ to first order we recover the SK Helmholtz free energy $N \ln 2+\beta^{2} / 4 \sum_{i, j} J_{i, j}^{2}$ because here $\sum_{i, j} J_{i, j}^{2}=N / 2+O(1)$ for $N$ large.

To obtain the Gibbs free energy we have to perform the Legendre transform

$$
\Phi\left(\beta, m_{i}\right) \equiv \max _{h_{i}}\left[F\left(\beta, h_{i}\right)-\sum_{i} h_{i} m_{i}\right]
$$

out of the $\left(h_{i}\right.$ dependent) expansion of (7) in powers of $\beta$. To this end we simply take over the Parisi-Potters argument because we are summing over a subclass of the "cactus" diagrams considered in [4] within the same assumption of self-averaging, 
namely $m_{k}^{2}=N^{-1} \sum_{l=1}^{N} m_{l}^{2}+o(1)$ as $N \rightarrow \infty$. This yields (5), whence the mean field equations (6) and the critical temperature.

It remains to prove that $N^{-1} \sum_{l=0}^{N-1} m_{k}^{l} \rightarrow 0$ as $N \rightarrow \infty$ if $J(A) m_{k}=m_{k}$. Any such eigenvector defines indeed a critical (staggered) magnetization distribution; furthermore the vanishing of the total magnetization represents the necessary condition both for the glassy nature of the transition as well as for self-averaging. Setting $\phi_{0}=$ $N^{-1 / 2}(1,1 \ldots, 1)$ we have $N^{-1} \sum_{l=0}^{N-1} m_{k}^{l}=\left\langle m_{k}, \phi_{0}\right\rangle=\left\langle m_{k}, V(A)^{l} \phi_{0}\right\rangle \forall l \in \mathbb{N}$. On the other hand [10] $V(A)$ tends (weakly) to a unitary operator on $L^{2}(-\pi, \pi)$ with Lebesgue spectrum on the unit circle as $N \rightarrow \infty$. This entails [15] $\lim _{l \rightarrow \infty} \lim _{N \rightarrow \infty}\left\langle m_{k}, V(A)^{l} \phi_{0}\right\rangle=$ $\lim _{N \rightarrow \infty}\left\langle m_{k}, \phi_{0}\right\rangle=0$.

Moreover by (5) the specific free energy $f=\beta \Phi / N$ is the same for all eigenvectors $m_{k}$ : the third term depends only on $q$, the second is $-\beta q / 2$ because $J m_{k}=m_{k}$ and

$$
2 N^{-1} \sum_{i=1}^{N}\left\{\left(1+m_{i}\right) \ln \left(1+m_{i}\right) / 2+\left(1-m_{i}\right) \ln \left(1-m_{i}\right) / 2\right\}=q+O\left(q^{3 / 2}\right)
$$

since $q$ is by definition small near the critical point. We conclude with two remarks.

(i) The staggered magnetizations can be explicitly computed for some particular values of $N$. There is indeed [10] $p(N) \in \mathbb{N}$ such that $J(A)^{p}=I_{d}$, and for "most" sequences of values of $N$ one has $p(N) / N=M, M<\infty$. Under these conditions the eigenvalue 1 of $J(A)$ has multiplicity $M+1$. A first corresponding (norm $\epsilon$ ) eigenvector is [9] $m_{1}=\cos (2 \pi i / N) \bar{k} l^{2} / \sqrt{\epsilon N}$ where $\bar{k}^{2}=3(\bmod N)$, while

$$
m_{l}^{r}=1 / \sqrt{\epsilon p} \sum_{s=0}^{p-1} c_{s}^{r} \cos (2 \pi i / N)\left(a_{s}^{r} l^{2}+b_{s}^{r} l\right) / \sqrt{N}, \quad r=2, \ldots, M+1
$$

where $\left|c_{s}^{r}\right| \leq 1$ and $0 \leq a_{s}^{r}, b_{s}^{r} \leq N-1$ are integers ( [9], Formula (4.25)). By (9) one checks directly that $N^{-1} \sum_{l=0}^{N-1} m_{l}^{r} \rightarrow 0$ for all eigenvectors.

(ii) The magnetization just below the critical point has the square-root behaviour of a second order transition. Compute indeed the second order expansion $\delta^{2} Q_{i}$ near $\beta_{c}$ and $m^{*}$ as in [5], putting $\beta=\beta_{c}+\Delta \beta$ and $m_{i}=m_{i}^{*}+\delta m_{i}$ where $m^{*}$ is any staggered 
magnetization vector and $\left\langle\delta m, m^{*}\right\rangle=0$. Neglecting the term of order $\Delta \beta^{2}$, taking the scalar product with $m^{*}$ and dividing by $N q$ the equation $\left\langle m^{*}, \delta^{2} Q\right\rangle=0$ we get $\alpha\|\delta m\|^{2}+\gamma \Delta \beta=0$ with $\alpha=-2 \beta_{c}^{3} G^{\prime \prime}\left(\beta_{c}\right)>0, \gamma=-1+2 G^{\prime}\left(\beta_{c}\right)+2 \beta_{c} G^{\prime \prime}\left(\beta_{c}\right)<0$, whence the assertion.

Acknowledgment. We thank G.Parisi for a useful conversation.

$1 \quad$ J.-P.Bouchaud And M.MÉzard, J.Phys.I (France) 4 (1994), 1109

2 E.Marinari, G.Parisi and F.Ritort, J.Phys.A (Math.Gen.) 27 (1994), 7615

3 E.Marinari, G.Parisi and F.Ritort, J.Phys.A (Math.Gen.) 27 (1994), 7647

4 G.PArisi And M.Potters, J.Phys.A (Math.Gen.) 28 (1995), 5267

5 J.Thouless, P.W.Anderson And T.Palmer, Phil.Mag. 35 (1977), 593

6 I.Borsari, S.Graffi And F.Unguendoli, J.Phys.A (Math.Gen.), to appear

7 J.Hannay And M.V.Berry, Physica D 1 (1980), 267

$8 \quad$ M.Degli Esposti, Ann.Inst.H.Poincaré 58 (1993), 7647

9 M.Degli Esposti, S.Graffi ANd S.Isola, Commun.Math.Phys. 167 (1995), 471

10 J.KeAting Nonlinearity 4 ( 1991), 309

11 D.R.Sherrington And A.L.KirckPAtrick, Phys.Rev.Letters 35, (1975), 1792

12 M.Mézard, G.PARisi And M.Virasoro, Spin Glass Theory and Beyond, World Scientific, Singapore (1985),

13 C.Itzykson, J.M.Drouffe, Statistical Field Theory, (Vol.II), Cambridge University Press, 1988 
14 T.Apostol, Introduction to Analytic Number Theory, Springer-Verlag, New York 1976

15 see e.g. I.P.Cornfeld, S.V.Fomin And YA.G.Sinai, Ergodic Theory, Springer-Verlag, New York 1986 